\title{
¿Por qué disminuyó la desigualdad en México? Análisis con los índices de Gini, Mehran y Piesch 2000-2012
}

\section{Why inequality declined in Mexico? Analysis with Gini, Mehran and Piesch Indexes, 2000-2012}

\author{
Genaro Aguilar-Gutiérrez*
}

\begin{abstract}
Using micro data from the National Household Income and Expenditure Survey, the methodology of decomposition of the Gini index of inequality, Mehran and Piesch shows that public programs ("Oportunidades") have contributed with $11.15 \%$ of the reduction in the observed inequality (Gini index) in Mexico between 2000 and 2012. The remittances received by households have contributed with $9.02 \%$ of the reduction of inequality. Mehran index shows that remittances contributed with $17.25 \%$ to the fall of the level of inequality.
\end{abstract}

Keywords: inequality, income distribution, Gini, Mehran and Piesch index decomposition.

\begin{abstract}
Resumen
En esta investigación se muestra -a partir de los cálculos con microdatos de la Encuesta Nacional de Ingresos y Gastos de los Hogares- con la metodología de descomposición de los índices de desigualdad de Gini, Mehran y Piesch, cómo los "programas públicos tipo Oportunidades" contribuyeron con 11,15\% a la reducción en la desigualdad observada (índice de Gini) en México entre 2000 y 2012. Las remesas que reciben los hogares explican al menos 9,02\% de la reducción en la desigualdad en el mismo periodo. El índice de Mehran muestra que las remesas contribuyeron con $17,25 \%$ a la caída del nivel de desigualdad.
\end{abstract}

Palabras clave: desigualdad, distribución del ingreso, descomposición de los índices de Gini, Mehran y Piesch.

\footnotetext{
${ }^{1}$ Este artículo es resultado del proyecto de investigación "Desarrollo Económico y Trabajo en México: Política Pública para generar trabajos de calidad contra la pobreza”, con registro número 20150483 de la Secretaría de Investigación y Posgrado del Instituto Politécnico Nacional

* Escuela Superior de Economía del Instituto Politécnico Nacional, México, correo-e: ggenaro 68mx@yahoo.com.br
} 


\section{Introducción}

La literatura sobre la relación entre crecimiento y pobreza tiene por objetivo identificar cuál debe ser el patrón de crecimiento económico y reducción de la desigualdad en un país o región considerado "ideal" para reducir la incidencia de la pobreza. Con respecto a la definición inicial de crecimiento pro-pobres, Kakwani y Pernia (2000) advirtieron que la reducción de la pobreza depende del crecimiento del ingreso promedio de la población y de cambios en su distribución; destacaron que una disminución en la desigualdad es necesaria para que el crecimiento pueda ser considerado pro-pobre. Los autores, al utilizar un método de descomposición proporcional en el cual se mantiene constante el componente distributivo, calcularon la variación total de la pobreza atribuida al componente crecimiento y, análogamente, midieron los efectos del componente distributivo, manteniendo constante el ingreso promedio de la sociedad.

Por otro lado, para medir cómo el crecimiento económico afecta la distribución del ingreso con el uso de microdatos, Ravallion y Chen (2003) propusieron la estimación de la curva de incidencia del crecimiento (growth incidence curve: GIC). La idea básica es el cálculo de la tasa de crecimiento en un determinado periodo para cada percentil de la distribución del ingreso. Así, los puntos de la GIC reflejan las tasas de crecimiento de las distribuciones acumuladas de ingreso -también llamada función cuantílica- para cada percentil. En una definición más restringida, si el crecimiento económico de una nación es pro-pobres, la pro-poor growth rate es mayor que la tasa de crecimiento ordinal del ingreso; es decir, el área bajo la GIC hasta el percentil definido por un índice de pobreza cualquiera debe ser mayor al punto final de la misma. Por lo que es necesario que la GIC tenga una inclinación negativa de dicha curva, lo cual es suficiente para definir un proceso de crecimiento económico como crecimiento pro-pobres; aun cuando la tasa media de crecimiento de toda la economía sea negativa para todos los percentiles del ingreso.

Los datos de la distribución del ingreso per cápita de los hogares en México muestran que dicho proceso, aunque marginal, se presentó en nuestro país entre 2000 y 2012; es decir, en México los hogares más pobres tuvieron un aumento relativo mayor que los más ricos en su participación en el ingreso total.

El objetivo de este trabajo es mostrar que una de las fuerzas propulsoras del incremento relativo del ingreso de los más pobres fueron las remesas que recibieron los hogares entre 2000 y 2012. Para ello, se realiza un análisis de descomposición de tres índices de desigualdad consagrados en la literatura: el clásico índice de Gini y los novedosos índices de 
Mehran y de Piesch. La descomposición de dichas medidas de desigualdad permite indagar si se presentó algún cambio estructural en el ingreso de las familias mexicanas por componentes de su ingreso y en qué medida ese cambio puede explicar la disminución de la desigualdad total.

En el primer apartado de esta investigación se muestra la curva de crecimiento para pobres, construida con datos de México para $2000 \mathrm{y}$ 2012. La segunda parte refiere la derivación matemática del proceso de descomposición de los índices de Gini, Mehran y de Piesch. En la tercera sección se presentan los resultados del cálculo de la desigualdad; así como los cálculos de la descomposición de los índices de desigualdad en México entre 2000 y 2012. Finalmente, se exponen las principales conclusiones del estudio.

\section{Evolución del ingreso de los más pobres}

Para cuantificar cómo el crecimiento económico (o la ausencia de crecimiento) afectó a los relativamente pobres, utilizaremos la "curva de crecimiento para pobres” propuesta por Son (2004). Sean $y_{1 h}$ así como $y_{2 h}$ los ingresos promedio de los $h \%$ más pobres en los dos años que aquí se compararán, 2000 y 2012. Para $h=30$, por ejemplo, tendríamos los ingresos promedio en los dos años comparados de los 30\% más pobres. Nótese que, para $h=100, y_{1 h}$ así como $y_{2 h}$ son los ingresos promedio de toda la población en cada año.

El crecimiento porcentual del ingreso promedio de los h\% más pobres en el periodo analizado es:

$$
r_{h}=\left(\frac{y_{2 h}}{y_{1 h}}-1\right) 100
$$

Se debe recordar que $r_{100}$ es el crecimiento del ingreso promedio de toda la población en el periodo considerado. El gráfico que muestra como $r_{b}$ varía en función de $h$, es la "curva de crecimiento para pobres" de Son (2004).

Para construir las curvas presentadas en la figura 1, los ingresos de 2000 y 2012 fueron deflactados a pesos de 1996. La figura 1 muestra las curvas de crecimiento para pobres en los periodos de 1996-2000; 20002012 y 1996-2012; la variable de análisis es el ingreso per cápita de los hogares en México.

En el periodo 1996-2000 la curva es creciente, lo cual significa que después de la crisis económica de 1995, el estilo de crecimiento de la economía favoreció proporcionalmente más a los estratos más ricos de la población. En los otros dos periodos (2000-2012 y 1996-2012) las 
curvas fueron decrecientes, mostrando que en esos periodos las variaciones en el ingreso fueron más favorables para los muy pobres (o relativamente más pobres). Como la ordenada del último punto de la curva del periodo 2000-2012 es negativa; esto significa que en ese periodo ocurrió una disminución del ingreso promedio del estrato más rico de la población: dicha caída fue de -11,09 por ciento.

Si analizamos lo que pasó con el ingreso promedio de los $40 \%$ más pobres en cada año, se verifica que creció $24,02 \%$ en el periodo 1996 a 2000; 26,73\% de 2000 a 2012, y 57,17\% de 1996 a 2012.

\section{Figura 1}

Curvas de crecimiento para pobres en los periodos 1996-2000, 2000-2012 y 1996-2012. México, ingreso per cápita de los hogares

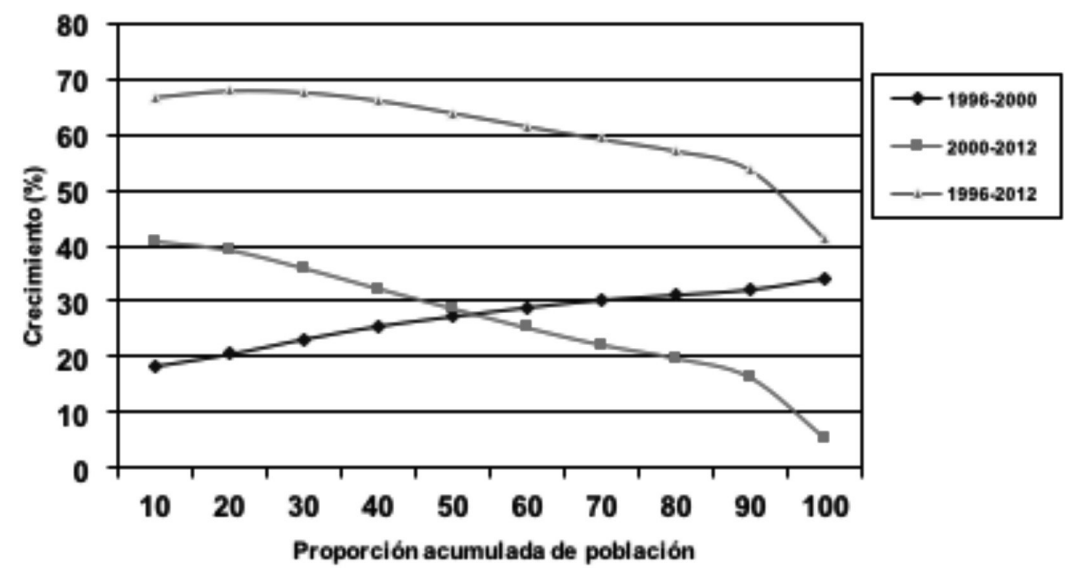

Fuente: elaboración propia a partir de microdatos de la Encuesta Nacional de Ingresos y Gastos de los Hogares 1996, 2000 y 2012.

Si la ordenada de la curva de crecimiento para pobres para $h<100$ es mayor que la ordenada del punto final (con $h>100)$, se puede concluir que se presentó una reducción de la desigualdad en el periodo analizado. La figura 1 muestra que en el periodo más largo, de 1996 a 2012, pero sobre todo en el subperiodo 2000-2012 hubo una reducción en la desigualdad en la distribución del ingreso per cápita de los hogares en México.

¿Cuáles son las fuerzas propulsoras de estos cambios distributivos? Recientemente un estudio de los cambios en la desigualdad en México fue realizado por Esquivel et al. (2010). Los autores realizaron un análisis de descomposición del índice de Gini concluyendo que la mejora de los ingresos de la población situada en el extremo inferior de la distribución (los más pobres) está explicada por el aumento de los salarios relativos de los trabajadores poco calificados, por el aumento en la proporción 
de las remesas hacia dichos estratos sociales y por la expansión de los programas de transferencias monetarias que realiza el gobierno federal hacia los más pobres.

Una explicación para la disminución de la desigualdad es el hecho de que la crisis económica, a partir del 2008, hizo que cayeran mucho los ingresos de los más ricos; en tanto que las familias más pobres aumentaron sus ingresos por dos vías: a) por las remesas enviadas a ellos por familiares en Estados Unidos y b) los ingresos de los extremadamente pobres subieron marginalmente debido al sistema de protección social mexicano, con "programas sociales tipo Oportunidades". Consecuentemente, la diferencia entre lo que recibieron los más ricos y lo que recibieron los más pobres disminuyó; haciendo que todos los índices de desigualdad cayeran.

Cowell (2008 y 1988) ha advertido, en literatura reciente y clásica sobre el tema, que el índice de Gini es muy sensible a movimientos en la parte "central" de la distribución y no precisamente en la "cola" inferior de la misma, es decir, es poco sensible a cambios distributivos que afecten a los extremadamente pobres. Por ello, entre las medidas novedosas más sensibles a cambios en el extremo inferior de la distribución destacan el índice de Mehran y, en el otro extremo del análisis, cambios en la parte superior de la distribución de los pobres pueden ser captados adecuadamente con el índice de Piesch.

Este trabajo analiza los cambios en la distribución del ingreso en México entre 2000 y 2012; para lo cual usamos los microdatos de la Encuesta Nacional de Ingresos y Gastos de los Hogares (ENIGH) para esos años y aplicamos el análisis de descomposición de los índices de Gini, Mehran y Piesch. La reducción en la desigualdad en la distribución del ingreso en México es lo que será analizado más cuidadosamente en las secciones 3 y 4; empleando la metodología que se presentará en la próxima sección.

\section{Método de descomposición de los índices de Gini, Mehran y Piesch}

\subsection{Descomposición del indice de Gini por fuentes de ingreso}

Desarrollos iniciales en la descomposición del índice de Gini mostraron, en la década de los setenta del siglo XX, que esa medida de desigualdad no debía ser descompuesta en las desigualdades dentro y entre los grupos; ya que hay un tercer término que capta el grado en el que los ingresos se superponen en los diferentes grupos; término que no podía ser aditivamente separado. Pyatt (1976), con su abordaje de juego estadístico para la descomposición de ese índice, contribuyó a una mejor comprensión de esa dificultad. Sin embargo, la descomposición del índice de Gini por 
factores siguió siendo analizada y desarrollada con herramientas matemáticas adicionales por otros como Pyatt et al., 1980; Shorrocks, 1980 y Cowell, 1988.

En 1980 Shorrocks hizo una severa crítica a la posibilidad de medir la contribución de componentes individuales de ingreso a la desigualdad total con los métodos usados en esa época. En su argumentación concluye que la mejor medida para ese propósito es la raíz cuadrada del coeficiente de variación; no obstante, es muy sensible a cambios en el extremo superior de la distribución; es decir, el coeficiente de variación como medida de desigualdad en la distribución del ingreso permite captar lo que ocurre cuando hay una transferencia de ingreso desde los ricos hasta los extremadamente ricos; sin embargo, es incapaz de captar adecuadamente cómo las transferencias regresivas de ingreso afectan a los más pobres.

A pesar de las críticas, diversos autores propusieron, desde la década de los ochenta del siglo XX, nuevas formas de descomponer el índice de Gini; por ejemplo, Shorrocks (1982) se basó en el cálculo de las razones de concentración por fuentes de ingreso. Investigaciones recientes con buenos resultados han aplicado la descomposición del índice de Gini partiendo de la propuesta inicial de Shorrocks, basada en la cuantificación de las razones de concentración de cada componente del ingreso total; entre ellas, las realizadas por Azevedo et al., 2013; Chantreuil y Trannov, 2013; Shorrocks, 2013; Aristondo et al., 2011; Chen et al., 2010; Hoffmann, 2009; Van, 2009; Griffiths, 2008; Cowell y Fiorio, 2006; Giovanni, 2006; Soares, 2006; Araar, 2004; Mussard et al., 2003, y Foster, et al., 2001.

El método de descomposición aplicado en este estudio es similar al analizado por Cowell y Fiorio (2006), el cual, en función de la disponibilidad de microdatos fue adaptado y aplicado recientemente por Hoffmann (2009) para el caso de Brasil; aquí seguiremos a este autor, quien muestra que el coeficiente de Gini puede ser fácilmente descompuesto en dos partes: el coeficiente de concentración de cada componente del ingreso en relación con el ingreso total y la proporción que cada componente tiene en el ingreso total.

A continuación presentaremos, pormenorizadamente, el procedimiento de descomposición de los cambios en el índice de Gini $(G)$.

Sea $x_{i}$ el ingreso familiar per cápita de la $i$-ésima persona, con $i=1,2$, $3, \ldots, n$. Esos ingresos están ordenados de manera que $x_{1}<x_{2}<x_{3}<\ldots<x_{n}$. Vamos a admitir que el ingreso $x_{i}$ está compuesto por $k$ componentes: 


$$
x_{i}=\sum_{h=1}^{k} x_{h i}
$$

Con los ingresos ordenados así, la curva de Lorenz muestra cómo la proporción acumulada de ingreso varía en función de la proporción acumulada de población. Siendo $\beta$ el área entre la curva de Lorenz y el eje de las abscisas, sabemos que el índice de Gini es:

$$
G=1-2 \beta
$$

Se puede demostrar que $0 \leq G<1$.

Si mantenemos el ordenamiento de los ingresos $x_{i}$, la curva de concentración de cada componente $x_{h i}$ muestra cómo la proporción acumulada de los $x_{h i}$ varía en función de la proporción acumulada de la población. Siendo $\beta_{b}$ el área entre esa curva y el eje de las abscisas, la razón de concentración de cada componente $x_{b i}$ es:

$$
C_{h}=1-2 \beta_{h}
$$

Se puede demostrar que $-1 \leq C_{b}<1$.

Si $\phi_{\mathrm{h}}$ es la participación del $h$-ésimo componente en el ingreso total, se puede demostrar que

$$
G=\sum_{h=1}^{k} \varphi_{h} C_{h}
$$

De esta forma, el índice de Gini de la distribución del ingreso puede ser dividido en $k$ componentes, correspondientes a las $k$ fracciones del ingreso per cápita de los hogares.

Consideremos ahora que el mismo tipo de descomposición del índice de Gini sea hecho en dos años distintos, indicados por los subíndices 1 y 2 :

$$
\begin{aligned}
G_{1} & =\sum_{h=1}^{k} \varphi_{1 h} C_{1 h} \\
G_{2} & =\sum_{h=1}^{k} \varphi_{2 h} C_{2 h}
\end{aligned}
$$


Entonces la variación del índice de Gini entre esos dos años es:

$$
\Delta G=G_{2}-G_{1}=\sum_{h=1}^{k}\left(\varphi_{2 h} C_{2 h}-\varphi_{1 h} C_{1 h}\right)
$$

Sumando y sustrayendo $\varphi_{1 h} \mathrm{C}_{2 h} \mathrm{y}$ factorizando, obtenemos:

$$
\Delta G=\sum_{h=1}^{k}\left(C_{2 h} \Delta \varphi_{h}+\varphi_{1 h} \Delta C_{h}\right)
$$

Con $\Delta \varphi_{h}=\varphi_{2 h}-\varphi_{1 h}$ y $\Delta C_{h}=C_{2 h}-C_{1 h}$

Alternativamente, sumando y substrayendo $\varphi_{2 h} \mathrm{C}_{1 h}$ dentro de la expresión entre paréntesis en (7) y factorizando, obtenemos:

$$
\Delta G=\sum_{h=1}^{k}\left(C_{1 h} \Delta \varphi_{h}+\varphi_{2 h} \Delta C_{h}\right)
$$

Las fórmulas (8) y (9) son dos maneras posibles de descomponer $\Delta \mathrm{G}$. Para evitar la cuestión de seleccionar arbitrariamente una de ellas, es razonable utilizar el promedio aritmético de las dos:

$$
\begin{aligned}
\Delta G=\sum_{h=1}^{k}\left(C_{h}^{*} \Delta \varphi_{h}+\varphi_{h}^{*} \Delta C_{h}\right) \\
C_{h}^{*}=\frac{1}{2}\left(C_{1 h}+C_{2 h}\right) \\
\varphi_{h}^{*}=\frac{1}{2}\left(\varphi_{1 h}+\varphi_{2 h}\right)
\end{aligned}
$$

El promedio de los índices de Gini en los dos años considerados sería:

$$
G^{*}=\frac{1}{2}\left(G_{1}+G_{2}\right)
$$

Por lo que se puede probar que

$$
\sum_{h=1}^{k} G^{*} \varphi_{h}=G^{*} \sum_{h=1}^{k}\left(\varphi_{2 h}+\varphi_{1 h}\right)=0
$$

Entonces la expresión (10) permanece con validez si sustraemos la ecuación (14) del segundo miembro, obteniendo

$$
\Delta G=\sum_{h=1}^{k}\left[\left(C_{h}^{k}-G^{*}\right) \Delta \varphi_{h}+\varphi_{h}^{*} \Delta C_{h}\right]
$$


Matemáticamente, tanto (10) como (15) son válidas; pero, cuando analizamos el significado económico de sus términos podemos concluir que (15) es la expresión correcta, como señala Soares (2006). En la ecuación (10), el aumento en la participación de un componente del ingreso $(\Delta \phi>0)$ sólo contribuye para reducir el índice de Gini si la respectiva razón de concentración $\left(C_{h}^{*}\right)$ es negativa. En la ecuación (15), el aumento en la participación de un componente del ingreso $(\Delta \phi>0)$ contribuye para la reducción del índice de Gini si la respectiva razón de concentración es menor que el índice de Gini $\left(C_{h}^{*}<G^{*}\right)$. De acuerdo con la fórmula (15), el aumento de la participación de un componente del ingreso $(\Delta \phi>0)$ contribuye para aumentar o disminuir el índice de Gini, en función de si la razón de concentración de ese componente del ingreso es mayor o menor que el índice de Gini, respectivamente.

Tomando la fórmula (15) como la descomposición del cambio en el índice de Gini, la contribución total del h-ésimo componente del ingreso para ese cambio es:

$$
(\Delta G)_{h}=\left(C_{h}^{*}-G^{*}\right) \Delta \varphi_{h}+\varphi_{h}^{*} \Delta C_{h}
$$

y la respectiva contribución porcentual es:

$$
s_{h}=\frac{100}{\Delta G}\left[\left(C_{h}^{*}-G^{*}\right) \Delta \varphi_{h}+\varphi_{h}^{*} \Delta C_{h}\right]
$$

En las ecuaciones (16) y (17) podemos distinguir un efecto asociado al cambio en la composición del ingreso, a lo que en la literatura especializada se le denomina efecto-composición (Chen, 2010; Griffiths, 2008; Cowell y Fiorio, 2006 y Soares, 2006) y un efecto asociado al cambio en las razones de concentración a lo que se denomina efecto-concentración. El efecto-composición del $h$-ésimo componente del ingreso es:

$$
\left(C_{h}^{*}-G^{*}\right) \Delta \varphi_{h}
$$

o, como porcentaje del cambio en el índice de Gini:

$$
s_{\varphi h}=\frac{100}{\Delta G}\left(C_{h}^{*}-G^{*}\right) \Delta \varphi_{h}
$$

Por lo tanto, el efecto-composición total es:

$$
\sum_{h=1}^{k}\left(C_{h}^{*}-G^{*}\right) \Delta \varphi_{h}
$$


A su vez, el efecto-concentración del $b$-ésimo componente del ingreso está dado por:

$$
\varphi_{h}^{*} \Delta C_{h}
$$

o, como porcentaje del cambio en el índice de Gini:

$$
s_{C h}=\frac{100}{\Delta G} \varphi_{h}^{*} \Delta C_{h}
$$

Y, por lo tanto, el efecto-concentración total es:

$$
\sum_{h=1}^{k} \varphi_{h}^{*} \Delta C_{h}
$$

En el siguiente apartado mostraremos la evolución reciente de la distribución del ingreso en México (2000 a 2012) y aplicaremos el proceso de descomposición del índice de Gini derivado anteriormente.

\subsection{Análisis formal de descomposición de los indices de Mehran $y$ de Piesch}

Como veremos en esta sección, el procedimiento de descomposición para los índices de Mehran $(M)$ y de Piesch $(P)$ es totalmente análogo al del índice de Gini $(G)$. De acuerdo con Sen y Singer (1994), los índices de Mehran y Piesch son casos particulares del coeficiente de Gini y fueron propuestos como medidas susceptibles de desagregarse aditivamente por los autores a quienes deben sus nombres. Para observar la semejanza de estas medidas con el índice de Gini, a continuación definiremos las tres medidas de desigualdad directamente asociadas con el área entre la curva de Lorez y la línea de plena equidad:

$$
\begin{array}{ll}
\text { Índice de Gini: } & G=\frac{2}{n} \sum_{i=1}^{n-1}\left(p_{i}-\phi_{i}\right), \\
\text { Índice de Mehran: } & M=\frac{6}{n} \sum_{i=1}^{n-1}\left(1-p_{i}\right)\left(p_{i}-\phi_{i}\right), \\
\text { Índice de Piesch: } & P=\frac{3}{n} \sum_{i=1}^{n-1} p_{i}\left(p_{i}-\phi_{i}\right),
\end{array}
$$

Donde $p_{i}$ y $\Phi_{i}$ son las coordenadas de los puntos en la curva de Lorenz y $n$ es el tamaño de la población.

En la fórmula (25) se puede observar que en el índice de Mehran la diferencia entre las coordenadas de la línea de plena equidad y las coor- 
denadas de la curva de Lorenz es ponderada por $\left(1-p_{i}\right)$; haciendo que esta medida de desigualdad sea relativamente más sensible a cambios en el extremo inferior de la distribución, es decir, en los primeros deciles o estratos de los más pobres, si la comparamos con el índice de Gini. En el índice de Piesch el factor de ponderación es $p_{i}$, haciendo que dicho índice sea más sensible a cambios que ocurren en el extremo superior de la distribución, es decir, en los últimos deciles o estratos de los más ricos.

Esta observación es relevante cuando se analiza la sensibilidad de las diversas medidas de desigualdad ante cambios en la distribución del ingreso; no todas reaccionan en la misma proporción ni, necesariamente, en la misma dirección. Lo deseable es que cuando una parte del ingreso de los más pobres es transferida a través de los mecanismos de mercado hacia el sector más adinerado de la población, las medidas de desigualdad crezcan, reflejando esa situación. De las medidas de desigualdad más difundidas (Theil, Redundancia de Theil, Atkinson, Gini, Mehran, Piesch) sin duda el índice de Gini es el que, de manera general, capta de una mejor manera los cambios en la desigualdad.

El índice de Gini es particularmente sensible a cambios que ocurren entre los deciles 3 a 7 de la distribución (Aguilar-Gutiérrez, 2012). Sin embargo, ante procesos de ingreso que siguen una distribución aproximadamente log-normal (es la distribución típica en las economías de mercado modernas), el índice de Mehran permite capturar de una mejor manera cambios en la distribución que afecten positiva o negativamente a los estratos de más bajos ingresos, que se encuentran en el extremo inferior de la distribución; es decir, a los más pobres entre los pobres. Por su parte, el índice de Piesch es más sensible a modificaciones en la distribución del ingreso que afectan a los estratos más ricos de la población.

El proceso de disminución en la desigualdad de la distribución del ingreso per cápita de los hogares que se presentó en México entre 2000 y 2012 sugiere cambios importantes en los dos extremos de la distribución. En función de ello, en esta investigación se aplica la descomposición de dichos cambios con los índices de Gini, Mehran y Piesch.

Usando la ponderación de factores $\left(1-p_{i}\right)$ del índice de Mehran, podemos definir las "razones de concentración" correspondientes a esta medida de desigualdad como:

$$
C_{M h}=\frac{6}{n} \sum_{i=1}^{n-1}\left(1-p_{i}\right)\left(p_{i}-\phi_{h i}\right)
$$

Análogamente, las razones de concentración asociadas con el índice de Piesch pueden ser calculadas con: 


$$
C_{P h}=\frac{3}{n} \sum_{i=1}^{n-1} p_{i}\left(p_{i}-\phi_{h i}\right)
$$

Es fácil verificar que:

$$
\begin{gathered}
M=\sum_{h \neq 1}^{k} \varphi_{h} C_{M h} \\
P=\sum_{h=1} \varphi_{h} C_{P h}
\end{gathered}
$$

Tenemos, así, una descomposición del índice de Mehran y del índice de Piesch análoga a la descomposición del índice de Gini. Son las fórmulas (16), (17), (19), (20), (22), (23), (29) y (30) las que se aplican en los cálculos de descomposición de la desigualdad en la distribución del ingreso per cápita de los hogares de México en el periodo 2000-2012 de la cuarta sección del presente estudio.

\section{Cambios en la desigualdad de la distribución del ingreso en México de 2000 a 2012}

En primer lugar presentaremos, sucintamente, la evolución de la desigualdad en México entre 2000 y 2012. Posteriormente desarrollaremos el análisis de la evolución de cada uno de los componentes del ingreso total y mostraremos los resultados del cálculo de la contribución de cada fuente de ingreso a la desigualdad total. Todos los cálculos se hicieron con las bases de microdatos de las Encuestas Nacionales de Ingresos y Gastos de los Hogares de esos años.

\subsection{Distribución 2000 vs. menor desigualdad en 2012}

El cuadro 1 muestra la distribución del ingreso per cápita de los hogares en México, en el 2000. La primera columna presenta los diferentes estratos de población ordenados en valores descendientes de ingreso. Así, 1\% superior se refiere a $1 \%$ de la población con ingreso más elevado; $5 \%$ comprende a $5 \%$ con ingreso más elevado, inclusive $1 \%$ superior. Enseguida tenemos la distribución por décimos de la población económicamente activa, comenzando, también, por los $10 \%$ más ricos y terminando con los $10 \%$ más pobres. La segunda columna muestra los porcentajes de ingreso correspondiente a cada grupo. La tercera y cuarta muestran, respectivamente, valores acumulados de los ingresos más altos hasta los más bajos y viceversa. La quinta columna muestra el ingreso promedio en dólares. Finalmente, las dos últimas columnas presentan los ingresos relativos de cada grupo, con relación a la mediana del ingreso (columna 6) y con relación al ingreso promedio (columna 7). En la parte 


\section{Cuadro 1}

\section{Distribución del ingreso per capita de los hogares en México en el 2000}

\begin{tabular}{|c|c|c|c|c|c|c|}
\hline \multirow[b]{3}{*}{$\begin{array}{c}\text { Grupo } \\
\text { delimitado por } \\
\text { percentil }\end{array}$} & \multicolumn{3}{|c|}{ Porcentaje de ingreso } & \multirow{3}{*}{$\begin{array}{l}\text { Ingreso } \\
\text { Promedio } \\
\text { en (US\$) }\end{array}$} & \multirow{2}{*}{\multicolumn{2}{|c|}{ Ingreso relativo }} \\
\hline & \multicolumn{3}{|c|}{ Acumulado } & & & \\
\hline & $\begin{array}{l}\text { En el } \\
\text { grupo }\end{array}$ & $\begin{array}{l}\text { A partir de } \\
\text { los ingresos } \\
\text { más altos }\end{array}$ & $\begin{array}{l}\text { A partir de } \\
\text { los ingresos } \\
\text { más bajos }\end{array}$ & & $\begin{array}{c}\text { con } \\
\text { relación a } \\
\text { la mediana }\end{array}$ & $\begin{array}{c}\text { con } \\
\text { relación al } \\
\text { promedio }\end{array}$ \\
\hline $1 \%$ superior & 13,97 & 13,97 & $86,03^{*}$ & 2371,41 & 25,13 & 13,97 \\
\hline $5 \%$ superior & 32,37 & 32,37 & $67,63^{*}$ & 1098,96 & 11,65 & 6,47 \\
\hline $10 \%$ superior & 44,82 & 44,82 & 100,00 & 760,82 & 8,06 & 4,48 \\
\hline 10 & 15,46 & 60,28 & 55,18 & 262,43 & 2,78 & 1,55 \\
\hline 10 & 10,46 & 70,74 & 39,72 & 177,56 & 1,88 & 1,05 \\
\hline 10 & 7,97 & 78,72 & 29,26 & 135,29 & 1,43 & 0,80 \\
\hline 10 & 6,34 & 85,05 & 21,28 & 107,62 & 1,14 & 0,63 \\
\hline 10 & 4,96 & 90,02 & 14,95 & 84,20 & 0,89 & 0,50 \\
\hline 10 & 3,90 & 93,92 & 9,98 & 66,20 & 0,70 & 0,39 \\
\hline 10 & 2,98 & 96,90 & 6,08 & 50,59 & 0,54 & 0,30 \\
\hline 10 & 2,04 & 98,94 & 3,10 & 34,63 & 0,37 & 0,20 \\
\hline $10 \%$ inferior & 1,06 & 100,00 & 1,06 & 17,99 & 0,19 & 0,11 \\
\hline
\end{tabular}

Índice de Gini: 0,558

Índice $\mathrm{T}$ de Theil: 0,483

Dual del T de Theil: 0,659

Mediana del ingreso: US\$94,36

Ingreso promedio: US $\$ 169,75$

Número total de personas: $98,310,615$

* Estos porcentajes se refieren al ingreso acumulado hasta los 99 y los $95 \%$ de la población, respectivamente. Así, los $1 \%$ más ricos reciben $13,97 \%$ del ingreso total y los $5 \%$ más ricos se apropian de $32,37 \%$ del ingreso nacional.

Fuente: elaborada a partir del procesamiento de los microdatos (datos hogar por hogar) de la Encuesta Nacional de Ingresos y Gastos de los Hogares 2000, Inegi, 2002.

inferior del cuadro están algunos índices de desigualdad (Gini, $T$ de Theil y su dual), bien como la mediana del ingreso y el ingreso promedio. Es importante notar que, por tratarse de la población en 2000, el total de personas era de alrededor de 98,3 millones de personas.

Se puede observar que el segmento constituido por $1 \%$ más rico de la población tenía $13,97 \%$ del total del ingreso, ${ }^{2}$ y tenía un ingreso promedio de 2,371 dólares mensuales, por persona. Considerando los $5 \%$ más ricos, la participación relativa se eleva a $32,37 \%$ y el ingreso promedio mensual cae hacia 1,099 dólares. El último décimo de la distribución (los

${ }^{2}$ El análisis que se desarrolla en este estudio toma como unidad de análisis a los hogares de México. El ingreso per cápita resulta de dividir el ingreso total entre el número de personas que habitan dichos hogares. Con la finalidad de tomar sólo el ingreso corriente, libre de fluctuaciones derivadas de la venta de activos; en este estudio se toma sólo el ingreso monetario y las percepciones de capital reportados en la Encuesta Nacional de Ingresos y Gastos de los Hogares. 
$10 \%$ más ricos) tenían $44,82 \%$ del total, con un ingreso promedio de 761 dólares por persona al mes. En el décimo siguiente se observa una caída brusca en la participación que es de $15,46 \%$ del ingreso total y el promedio cae hasta 262 dólares.

Después de ese décimo, las reducciones en las participaciones de los diferentes grupos son menores, sugiriendo una mayor desigualdad entre los estratos más ricos si la comparamos con la que existe entre los grupos más pobres de la población (a pesar de que los 10\% más pobres reciben apenas 17,99 dólares por mes, por habitante; mientras que el décimo siguiente tiene ingreso promedio $92,5 \%$ mayor, de 34,63 dólares mensuales).

Es importante notar la magnitud de la diferencia entre la mediana del ingreso (94,36 dólares) y el ingreso promedio (169,75 dólares), que indica una fuerte asimetría positiva en la distribución. Así, el ingreso relativo de $1 \%$ superior varía de 25 a 13, según sea hecha la comparación con relación a la mediana del ingreso o con relación al ingreso promedio. Por definición, los $50 \%$ más pobres de la población tienen percepciones inferiores a la mediana del ingreso y, por indagación, los $70 \%$ más desfavorecidos tienen ingreso menor al promedio.

¿Qué ocurrió con la distribución del ingreso en 12 años? El cuadro 2 permite observar cómo los ingresos de los deciles más pobres creció relativamente más su participación en el ingreso total de los hogares; debido a lo cual el índice de Gini que en el 2000 era de 0,558 (ver cuadro 1); bajó a 0,501 en el 2012 (cuadro 2). Efectivamente: el decil más pobre tenía un ingreso que representaba $1,06 \%$ del ingreso total en el 2000 y pasó a 1,25\% en 2012. El segundo decil que tenía 2,04\% pasó a 2,61\%; el tercero tenía $2,98 \%$ y subió a 3,64\% y, finalmente, el cuarto decil pasó de 3,90\% a 4,61\% del ingreso total del 2000 a 2012.

El cuadro 2 muestra la distribución del ingreso per cápita de los hogares en México en 2012; en él se describe que 1\% de la población más rica detentaba $10,85 \%$ del ingreso total y tenía un ingreso promedio de 2,222 dólares por mes. Los $5 \%$ más ricos recibieron $27,13 \%$ del ingreso total, con un ingreso promedio mensual de 1,111 dólares. Los $10 \%$ más ricos tenían 39,06\% del total, con un ingreso promedio de 800 dólares. En el décimo siguiente se observa una caída importante en la participación, pasando a $16,0 \%$ del total, con un ingreso promedio de 328 dólares.

Después de ese décimo, las reducciones en las participaciones de los diferentes grupos son menores, sugiriendo nuevamente una mayor desigualdad entre los estratos más ricos. Las diferencias en las medidas de desigualdad entre las dos distribuciones analizadas son significativas: la participación de los $10 \%$ más ricos cae de $44,82 \%$ a $39,06 \%$, con lo que el índice de Gini bajó, como habíamos visto, de 0,558 a 0,501 (una caída de 11,37 por ciento). 


\section{Cuadro 2}

\section{Distribución del ingreso per cápita de los hogares en México en 2012}

\begin{tabular}{|c|c|c|c|c|c|c|}
\hline \multirow[b]{3}{*}{$\begin{array}{c}\text { Grupo } \\
\text { delimitado por } \\
\text { percentil }\end{array}$} & \multicolumn{3}{|c|}{ Porcentaje de ingreso } & \multirow{3}{*}{$\begin{array}{c}\text { Ingreso } \\
\text { Promedio } \\
\text { en (US\$) }\end{array}$} & \multirow{2}{*}{\multicolumn{2}{|c|}{ Ingreso relativo }} \\
\hline & & Acum & ulado & & & \\
\hline & $\begin{array}{l}\text { En el } \\
\text { grupo }\end{array}$ & $\begin{array}{l}\text { A partir de } \\
\text { los ingresos } \\
\text { más altos }\end{array}$ & $\begin{array}{c}\text { A partir de } \\
\text { los ingresos } \\
\text { más bajos }\end{array}$ & & $\begin{array}{c}\text { con } \\
\text { relación a } \\
\text { la mediana }\end{array}$ & $\begin{array}{c}\text { con } \\
\text { relación al } \\
\text { promedio }\end{array}$ \\
\hline $1 \%$ superior & 10,85 & 10,85 & $89,15^{*}$ & 2221,79 & 17,07 & 10,85 \\
\hline $5 \%$ superior & 27,13 & 27,13 & $72,87^{*}$ & 1111,10 & 8,54 & 5,43 \\
\hline $10 \%$ superior & 39,06 & 39,06 & 100,00 & 799,84 & 6,15 & 3,91 \\
\hline 10 & 16,00 & 55,05 & 60,95 & 327,64 & 2,52 & 1,60 \\
\hline 10 & 11,34 & 66,39 & 44,95 & 232,21 & 1,78 & 1,13 \\
\hline 10 & 8,74 & 75,14 & 33,61 & 178,97 & 1,38 & 0,87 \\
\hline 10 & 7,02 & 82,16 & 24,87 & 143,75 & 1,10 & 0,70 \\
\hline 10 & 5,72 & 87,88 & 17,84 & 117,13 & 0,90 & 0,57 \\
\hline 10 & 4,61 & 92,49 & 12,12 & 94,40 & 0,73 & 0,46 \\
\hline 10 & 3,64 & 96,13 & 7,51 & 74,54 & 0,57 & 0,36 \\
\hline 10 & 2,61 & 98,75 & 3,87 & 53,45 & 0,41 & 0,26 \\
\hline $10 \%$ inferior & 1,25 & 100,00 & 1,25 & 25,60 & 0,20 & 0,12 \\
\hline
\end{tabular}

Índice de Gini: 0,501

Índice $T$ de Theil: 0,394

Dual del T de Theil: 0,500

Mediana del ingreso: US\$130,15

Ingreso promedio: US $\$ 204,77$

Número total de personas: $112,600,583$

Fuente: construida a partir del procesamiento de los microdatos (datos hogar por hogar) de la Encuesta Nacional de Ingresos y Gastos de los Hogares 2012; Inegi, 2013.

\subsection{Distribución y concentración de los ingresos de los hogares en México}

El ingreso monetario de los hogares en México puede ser dividido, grosso modo, en seis componentes:

a) Ingreso de todos los trabajos, incluyendo sueldos, salarios y remuneración de los trabajadores por cuenta propia y patrones; variable que denominaremos genéricamente salarios.

b) Ingresos por negocios, sin importar si se trata de empresas de carácter familiar, si tienen o no empleados, si son micro, pequeñas o medianas empresas. En la ENIGH aparecen clasificados como "ingresos por negocios". 

c) Ingresos por rentas. ${ }^{3}$
d) Ingresos por transferencias (incluye pensiones, remesas y beneficios de "programas sociales o públicos tipo Oportunidades").
e) Otros ingresos monetarios.
f) Ingresos de capital.

En el cuadro 3 están las participaciones $\left(\phi_{\mathrm{h}}\right)$ de cada componente en el ingreso total de los hogares de México en 2000 y 2012. Se observa que en ese periodo la participación de los salarios sube de $56,21 \%$ a $64,87 \%$, la participación de los ingresos por negocios cae de $23,49 \%$ a $10,72 \%$. El rubro de ingresos por rentas sube de $1,36 \%$ a 4,38 por ciento.

\section{Cuadro 3 \\ Participación de cada componente en el ingreso total de los hogares en México, 2000 y 2012}

\begin{tabular}{llll}
\hline \multicolumn{1}{c}{ Componente } & 2000 & 2012 & Crecimiento \\
\hline Salarios & 0,5621 & 0,6487 & 0,0866 \\
Ingresos por negocios & 0,2349 & 0,1072 & $-0,1277$ \\
Rentas & 0,0136 & 0,0438 & 0,0302 \\
Transferencias & 0,1070 & 0,1446 & 0,0376 \\
Pensiones & 0,0427 & 0,0753 & 0,0326 \\
Remesas & 0,0582 & 0,0403 & $-0,0179$ \\
Oportunidades & 0,0060 & 0,0290 & 0,0230 \\
Otros ingresos & 0,0006 & 0,0011 & 0,0005 \\
Ingresos de capital & 0,0818 & 0,0546 & $-0,0272$ \\
\hline
\end{tabular}

Fuente: cálculos a partir de los microdatos (datos hogar por hogar) de la Encuesta Nacional de Ingresos y Gastos de los Hogares (Inegi, 2000 y 2012). En el 2000 el rubro de Remesas estaba cuantificado dentro del rubro genérico de donativos; y el de Oportunidades en el rubro de beca-donativos; en el 2012: los "programas tipo Oportunidades" están clasificados como beneficios gubernamentales.

La participación de las transferencias (incluyendo pensiones, remesas desde el extranjero y programas sociales) aumenta su participación en el ingreso de los hogares al pasar de 10,70\% a 14,46\%, el rubro de "programas públicos tipo Oportunidades" ${ }^{\prime 4}$ aumenta de $0,6 \%$ a 2,9\%. Las percepciones de capital bajan de $8,18 \%$ a 5,46 por ciento.

${ }^{3}$ La Encuesta Nacional de Ingresos y Gastos de los Hogares establece que "La renta de la propiedad agrupa todos los ingresos que reciben los integrantes del hogar derivados de la posesión de activos financieros o tangibles que han puesto a disposición de otras unidades".

${ }^{4}$ En la base de microdatos de la ENIGH, los ingresos de los hogares derivados de programas públicos se denominan, genéricamente, "Beneficios gubernamentales". En este concepto se capturan todos los ingresos de los hogares derivados de los siguientes programas públicos (con clave de ingreso): P042. Beneficio de "Oportunidades"; P043. Beneficio de "Procampo"; P044. Beneficio del programa "70 y más"; P045. Beneficio de otros programas para adultos mayores; P046. Beneficio del programa Alimentario; P047. Beneficio del programa Empleo Temporal; y P048. Beneficios de otros 
El cuadro 4 muestra las razones de concentración $\left(C_{h}\right)$ en relación con el índice de Gini; en la última línea aparece el valor del propio índice en cada año. Se puede observar la tendencia a disminuir en el índice que pasa de 0,5575 en el 2000 a 0,5011 en el 2012; una caída de 10,12 por ciento.

La razón de concentración de los salarios cae de 0,5204 a 0,4958, y tanto en el 2000 como en 2012 es inferior al índice de Gini. Esto significa que los salarios se distribuyen más equitativamente que el ingreso total de los hogares en México. Paradójicamente, como veremos más adelante, aun así los salarios tienen un impacto relativamente pequeńo en la disminución de la desigualdad en México.

La razón de concentración para pensiones es significativamente superior al índice de Gini en ambos años, lo que muestra que la distribución de las pensiones está reforzando el elevado grado de desigualdad en la distribución del ingreso en México. El "componente-Oportunidades", captado como un subcomponente de las transferencias oficiales, tiene una distribución más equitativa que el ingreso nacional; y aún más: las razones de concentración negativas para este componente del ingreso están mostrando que dicha fuente de ingresos beneficia más a los segmentos de población con más bajos ingresos (gráficamente estaría siendo representado con una curva de Lorenz invertida). Esto puede ser interpretado como un interesante resultado de la política social en México: estos programas han sido focalizados eficientemente hacia los ciudadanos más pobres.

La razón de concentración de las remesas (el dinero que los miembros de los hogares reciben de sus familiares que trabajan en Estados Unidos) es siempre inferior al índice de Gini. Más aún, la brecha que existía entre el índice de Gini y la razón de concentración de las remesas aumentó pasando de 0,1879 en el 2000 a 0,2321 en el 2012. Como veremos más adelante al medir el efecto de las remesas sobre la desigualdad total, esto significa que las remesas desempeñan un papel relevante y creciente en la disminución de la desigualdad en la distribución del ingreso per cápita entre los hogares de México.

El cambio más importante que se puede observar en el cuadro 4 es la caída en la razón de concentración de los ingresos por negocios: en el 2000 esa razón era de 0,5740 y en el 2012 bajó a 0,4156. Así, al final del periodo de estudio el ingreso derivado de los negocios se ha distribuido más equitativamente. Debe indicarse aquí que la clasificación de la Encuesta Nacional de Ingresos y Gastos de los Hogares establece como ingresos por negocios a todas aquellas entradas en efectivo o en especie que

programas sociales. En este estudio decidimos no desagregar el ingreso de los hogares en cada uno de esos rubros; sino llamarlos a todos, genéricamente, "Programas públicos tipo Oportunidades". 


\section{Cuadro 4}

Razones de concentración $\left(C_{b}\right)$ en relación con el índice de Gini de la distribución del ingreso per cápita de los hogares en México, 2000 y 2012

\begin{tabular}{lccr}
\hline \multicolumn{1}{c}{ Componente } & 2000 & 2012 & Crecimiento \\
\hline Salarios & 0,520364 & 0,495811 & $-0,0246$ \\
Ingresos por negocios & 0,574005 & 0,415607 & $-0,1584$ \\
Rentas & 0,801449 & 0,784223 & $-0,0172$ \\
Transferencias & 0,496278 & 0,432138 & $-0,0641$ \\
Pensiones & $\mathbf{0 , 6 8 5 1 0 7}$ & $\mathbf{0 , 6 7 7 7 4 3}$ & $\mathbf{- 0 , 0 0 7 4}$ \\
Remesas & $\mathbf{0 , 3 6 9 5 9 4}$ & $\mathbf{0 , 2 6 8 9 9 6}$ & $\mathbf{- 0 , 1 0 0 6}$ \\
Oportunidades & $\mathbf{- 0 , 1 6 9 8 2 0}$ & $\mathbf{- 0 , 2 2 4 3 3 1}$ & $\mathbf{- 0 , 0 5 4 5}$ \\
Otros ingresos & 0,385900 & 0,425993 & 0,0401 \\
Ingresos de capital & 0,815587 & 0,691402 & $-0,1242$ \\
Índice de Gini & 0,557525 & 0,501103 & $-0,0570$ \\
\hline
\end{tabular}

Fuente: cálculos a partir de los microdatos (datos hogar por hogar) de la Encuesta Nacional de Ingresos y Gastos de los Hogares (Inegi, 2000 y 2012).

los integrantes del hogar reciben regularmente por su desempeño como trabajadores independientes en su trabajo principal o, si es el caso, en su trabajo secundario.

Esto significa que el ingreso de cualquier persona que se dedique a desarrollar labores en forma independiente o en sociedad (puede ser un jardinero, un plomero, un trabajador de una empresa que a su vez tenga un local afuera de su casa para vender cualquier tipo de mercancía; una vendedora de alimentos o un profesionista por cuenta propia; pero también un productor agrícola o un gran empresario) es considerado, bajo esta clasificación, como ingresos por negocios.

En la ENIGH las "ganancias" de una persona independiente se clasifican de igual forma que las de una persona que tenga un negocio relativamente grande y consolidado. Pues bien, los resultados indican que este tipo de ingresos tendió a concentrarse menos y a distribuirse más equitativamente entre los diferentes grupos sociales. Por lo tanto, investigaciones posteriores deberán indagar si se presentó un aumento importante en el número de negocios o empresas para los deciles más pobres de la población. Quizás en el periodo analizado hayan crecido el número de negocios por cuenta propia en manos de los segmentos más pobres de la sociedad mexicana, por lo que la razón de concentración de los ingresos por negocios cayó en más de 27,59 por ciento. 
Por otro lado, la razón de concentración de las percepciones de capital cae de 0,8156 (altamente concentradas) a 0,6914 (aún altamente concentradas, pero menos que en 2010). Algún mecanismo económico está llevando a que los ingresos de capital se distribuyan "hacia abajo" en la estructura de los deciles de los hogares en México. Volveremos sobre esta cuestión en la sección 3.4. de este estudio.

Los cuadros 5 y 6 muestran para cada componente del ingreso per cápita de los hogares en México, las razones de concentración con relación a los índices de Mehran y Piesch; respectivamente, para 2000 y 2012. La última línea de cada cuadro muestra los valores del respectivo índice en cada año. En el cuadro 5 podemos observar que como un todo, el índice de Mehran muestra (al igual que el Gini) que la desigualdad en la distribución del ingreso per cápita de los hogares de México cayó entre 2000 y 2012: el índice pasa de 0,6759 a 0,5954; una caída de 11,92\%. La razón de concentración para los salarios que era inferior al índice en el 2000, cayó ligeramente, pero el índice cayó más; de tal forma que la razón de concentración de los salarios quedó, en 2012, superior al valor del índice de Mehran.

El hecho de que el índice de Mehran sea más sensible a movimientos en el extremo inferior de la distribución hace que el aumento de la razón de concentración para salarios, con este índice, indique que los salarios se concentraron más del 2000 al 2012: los más ricos ganaron en el 2012 más por salarios, que lo que ganaban en relación con los más pobres por el mismo concepto, doce años antes. También se puede interpretar como un indicador de que los salarios de los altos ejecutivos de las empresas o del gobierno crecen más rápidamente que los salarios de las personas más pobres.

Algo en sentido contrario ocurrió con el ingreso por negocios: la razón de concentración de este componente pasó de 0,7059 (mayor al índice de Mehran) en 2000; a 0,5158 (menor que el índice de Mehran) en el 2010. Al igual que con el índice de Gini, estos datos indican que los más pobres tuvieron un mayor acceso a ingresos por negocios en el 2012 que en el 2000.

La razón de concentración de las percepciones de capital, para este índice eran superiores al propio valor del índice en el 2000 y pasaron a ser inferiores al índice de Mehran en el 2012; lo que lleva a la conclusión de que las percepciones de capital "se democratizaron" en el sentido de que aquellos sectores de la población más pobres tuvieron, de alguna forma, acceso a mayores ingresos por este concepto. 


\section{Cuadro 5}

Razones de concentración $\left(C_{b}\right)$ en relación con el índice de Mehran de la distribución del ingreso per cápita de los hogares en México, 2000 y 2012

\begin{tabular}{lccr}
\hline \multicolumn{1}{c}{ Componente } & 2000 & 2012 & Crecimiento \\
\hline Salarios & 0,63799 & 0,61259 & $-0,02540$ \\
Ingresos por negocios & 0,70596 & 0,51576 & $-0,19020$ \\
Rentas & 0,95049 & 0,93739 & $-0,01310$ \\
Transferencias & 0,60028 & 0,51959 & $-0,08069$ \\
Pensiones & 0,78004 & 0,77204 & $-0,00800$ \\
Remesas & 0,41053 & 0,18529 & $-0,22524$ \\
Oportunidades & $-0,20193$ & $-0,25058$ & $-0,04866$ \\
Otros ingresos & 0,45961 & 0,50222 & 0,04261 \\
Ingresos de capital & 0,90593 & 0,47626 & $-0,42966$ \\
Índice de Mehran & 0,67598 & 0,59542 & $-0,08056$ \\
\hline
\end{tabular}

Fuente: cálculos a partir de los microdatos (datos hogar por hogar) de la Encuesta Nacional de Ingresos y Gastos de los Hogares (Inegi, 2000 y 2012).

\section{Cuadro 6}

Razones de concentración $\left(C_{b}\right)$ en relación con el índice de Piesch de la distribución del ingreso per cápita de los hogares en México, 2000 y 2012

\begin{tabular}{lccc}
\hline \multicolumn{1}{c}{ Componente } & 2000 & 2012 & Crecimiento \\
\hline Salarios & 0,4615 & 0,4374 & $-0,0241$ \\
Ingresos por negocios & 0,5080 & 0,3656 & $-0,1425$ \\
Rentas & 0,7269 & 0,7076 & $-0,0193$ \\
Transferencias & 0,4443 & 0,3883 & $-0,0560$ \\
Pensiones & 0,6376 & 0,6306 & $-0,0070$ \\
Remesas & 0,3491 & 0,3108 & $-0,0383$ \\
Oportunidades & $-0,1604$ & $-0,2004$ & $-0,0400$ \\
Otros ingresos & 0,3494 & 0,4010 & 0,0516 \\
Ingresos de capital & 0,7248 & 0,7990 & 0,0742 \\
Índice de Piesch & 0,4957 & 0,4541 & 0,0415 \\
\hline
\end{tabular}

Fuente: cálculos a partir de los microdatos (datos hogar por hogar) de la Encuesta Nacional de Ingresos y Gastos de los Hogares (Inegi, 2000 y 2012).

Es interesante notar que las razones de concentración de los ingresos por transferencias son siempre menores que el índice de Mehran. Este resultado está indicando; como ocurrió con el índice de Gini, que las transferencias (con excepción de las pensiones) desempeñan un papel importante en la reducción de las desigualdades. La razón de concentración de las pensiones es significativamente superior al valor del índice, lo 
que indica que dicho concepto actúa profundizando la desigualdad. Sin embargo, la razón de concentración tanto de las remesas como de los "programas tipo Oportunidades" son inferiores al valor del índice; lo cual significa que estos conceptos actúan favoreciendo una menor desigualdad en la distribución del ingreso de los hogares de México.

El cuadro 6 muestra las razones de concentración en relación con el índice de Piesch. La razón de concentración de los salarios es siempre un poco menor que el índice de Piesch (recordemos, sin embargo, que esta medida es más sensible a modificaciones en la parte superior de la distribución). Nuevamente, la razón de concentración de los ingresos por negocios que era superior al índice en el 2000, cae en forma relevante, siendo inferior en el 2012 al valor del índice de Piesch. La razón de concentración para las transferencias es inferior al valor de su índice, indicando que ese componente del ingreso contribuye a la disminución de la desigualdad como un todo. Sin embargo, nuevamente se observa, en forma destacada, que las pensiones tienen una razón de concentración superior al índice de Piesch, tanto en el 2000 como en el 2012; más aún: las pensiones que ya tenían una razón de concentración, 22,25\% superior al índice en el 2000, aumentaron dicho proceso de concentración tornándose $27,99 \%$ más concentrados que el ingreso total en el 2012. Esto lleva a la conclusión de que las pensiones son un factor que eleva la desigualdad con que se distribuyen los ingresos en México.

Por último, la razón de concentración de las remesas y de los "programas tipo Oportunidades" son inferiores al índice de Piesch. Lo que indica que estos dos componentes del ingreso de los hogares están actuando como factores que ayudan a disminuir la desigualdad en la distribución del ingreso.

Las fórmulas derivadas del análisis matemático de la sección 3 pueden ser aplicadas para medir el efecto que cada componente del ingreso de los hogares de México tiene en la desigualdad total en la distribución del ingreso a partir de las participaciones y de las razones de concentración de esas fuentes de ingreso para cada medida de desigualdad; eso es lo que presenta la sección 4.3 .

\subsection{Descomposición de los cambios en la desigualdad 2000-2012}

Del cuadro 3 (participaciones) y de los cuadros 4, 5 y 6 del apartado anterior podemos descomponer los efectos de cada componente del ingreso en efecto-composición y efecto-concentración, así como medir el efecto porcentual de cada componente sobre la variación de cada medida de desigualdad. 
Vamos a examinar, en primer lugar, la descomposición del cambio en el índice de Gini del 2000 al 2012. En ese periodo, el índice de Gini de la distribución del ingreso per cápita de los hogares en México cayó de 0,5575 a 0,5011; por lo que el $\Delta G=-0,0570$. El cuadro 7 muestra la descomposición de esa variación de acuerdo con las fórmulas (17), (19) y (22); distinguiendo el efecto-composición y el efecto-concentración. Podemos observar que el efecto-concentración total representa $103,37 \%$ de $\Delta G$. Para varios componentes de ingreso el efecto-concentración tiene un valor absoluto mayor que el efecto-composición, la excepción son los ingresos por rentas, pensiones y por "programas públicos tipo Oportunidades".

En el cuadro 7 están las contribuciones de cada componente del ingreso a la variación del índice de Gini entre 2000 y 2012. El índice de Gini cayó en aproximadamente 11\% y más de 39\% de esa reducción está asociada al ingreso por negocios: parece cada vez más claro que una modificación estructural en la economía mexicana ha permitido que sectores cada vez más pobres de la sociedad tengan acceso a ingresos por negocios familiares.

\section{Cuadro 7}

Descomposición del cambio en el índice de Gini de la distribución del ingreso per cápita de los hogares en México, 2000 y 2012: $\Delta G$

\begin{tabular}{lrrr}
\hline \multicolumn{1}{c}{ Componente } & $\begin{array}{r}\text { Efecto-composición } \\
\% \text { de } \Delta G\end{array}$ & $\begin{array}{r}\text { Efecto-concentración } \\
\text { \% de } \Delta G\end{array}$ & $\begin{array}{c}\text { Total } \\
\% \text { de } \Delta G\end{array}$ \\
\hline Salarios & 3,29 & 26,06 & 29,35 \\
Ingreso por negocios & $-7,83$ & 47,50 & 39,68 \\
Rentas & $-13,93$ & 0,87 & $-13,06$ \\
Transferencias & 4,32 & 14,15 & 18,47 \\
Pensiones & $-2,67$ & 0,97 & $-1,70$ \\
Remesas & $-2,03$ & 11,05 & 9,02 \\
Oportunidades & 9,02 & 2,13 & 11,15 \\
Otros ingresos & 0,11 & $-0,06$ & 0,05 \\
Ingresos de capital & 10,67 & 14,85 & 25,52 \\
Total & $-3,37$ & 103,37 & 100,00 \\
Cambio en el índice de Gini & & & $-0,05703746$ \\
\hline
\end{tabular}

Fuente: cálculos a partir de los microdatos (datos hogar por hogar) de la Encuesta Nacional de Ingresos y Gastos de los Hogares (Inegi, 2000 y 2012).

La segunda mayor contribución a la reducción del índice de Gini está asociada al ingreso por salarios (29,35\%). Sin embargo, recordando los datos del cuadro 3, que muestran que los salarios aumentaron su participación en el ingreso total de los hogares de 56,21\% en el 2000 a 64,87\% en el 2012; se esperaría que por esa única razón los salarios contribuyeran 
en forma muy relevante a disminuir la desigualdad, y ello no está ocurriendo. La única explicación de esta situación radica en el hecho de que a medida que pasa el tiempo, los ingresos por sueldos y salarios de los deciles más ricos de la población están creciendo más rápidamente que los ingresos por el mismo concepto para los deciles más pobres, como veremos en la sección 4.4 .

En esta parte del análisis queremos destacar y llamar la atención sobre el papel que desempeñan en la disminución de la desigualdad, dos componentes del ingreso de los hogares: el rubro de "Ingreso por programas tipo Oportunidades" así como "Ingresos por Remesas". A pesar de que estas fuentes del ingreso tienen una muy pequeña participación en el ingreso total de los hogares (4,0\% para remesas y 2,9\% para Oportunidades en el 2012); el efecto de ellas es muy relevante para la disminución de la desigualdad total: el efecto de los "programas tipo Oportunidades" es de $11,15 \%$ en la disminución del Gini total. El efecto de las remesas es de 9,02\% para disminuir el Gini del ingreso per cápita de los hogares en México en el periodo 2000-2012 (ver cuadro 7).

A pesar de algunas críticas recientes, una primera importante conclusión de nuestros resultados es que, a juzgar por los resultados en la descomposición del índice de Gini, el programa de transferencias monetarias condicionadas "Oportunidades" ha tenido un efecto significativo en la mejoría relativa de los hogares más pobres de nuestro país. Levy (2010), al criticar los resultados de este programa, afirma que "Oportunidades" ha generado estímulos en la población pobre para mantenerse en la informalidad. Estos resultados deberían tomarse en cuenta en la redefinición de los programas públicos en México: en 2014 el "Programa Oportunidades" se transformó en "Prospera", para incluir un componente productivo a la estrategia.

Por lo tanto, se verifica que dos componentes de las transferencias: los ingresos por Remesas y por los "programas públicos tipo Oportunidades" han tenido un efecto relevante para disminuir las desigualdades en México: entre ambos componentes explican 20\% de la caída del índice de Gini.

El componente Pensiones influye negativamente, haciendo que la desigualdad crezca en 1,7\%. De igual forma, los ingresos por rentas tienen un efecto negativo, provocan que la desigualdad crezca. En todas las economías el "efecto rentas" suele ser un factor de aumento de la desigualdad; esto es lógico si tomamos en cuenta que las rentas se derivan fundamentalmente de los activos físicos acumulados por los hogares y, en hogares ricos esa acumulación es más probable que en los pobres.

En los cuadros 8 y 9 se pueden observar los efectos sobre los cambios en los índices de Mehran y de Piesch, de cada componente del ingreso de los hogares. Se puede concluir: 


\section{Cuadro 8}

Efecto porcentual de cada componente del ingreso en el cambio del índice de Mehran $(M)$ de la distribución del ingreso per cápita de los hogares en México, 2000-2012

\begin{tabular}{lr}
\hline \multicolumn{1}{c}{ Componente } & Efecto \\
\hline Salarios & 28,66 \\
Ingreso por negocios & 34,62 \\
Rentas & $-16,06$ \\
Transferencias & 30,49 \\
Pensiones & $\mathbf{- 1 , 6 2}$ \\
Remesas & $\mathbf{1 7 , 2 5}$ \\
Oportunidades & $\mathbf{1 4 , 8 7}$ \\
Otros ingresos & 0,00 \\
Ingresos de capital & 22,29 \\
Total & 100,00 \\
Cambio en el índice de Mehran & $-0,0805584$ \\
\hline
\end{tabular}

Fuente: cálculos a partir de los microdatos (datos hogar por hogar) de la Encuesta Nacional de Ingresos y Gastos de los Hogares (Inegi, 2000 y 2012).

\section{Cuadro 9}

Efecto porcentual de cada componente del ingreso en el cambio del índice de Piesch $(P)$ de la distribución del ingreso per cápita de los hogares en México, 2000-2012

\begin{tabular}{lc}
\hline \multicolumn{1}{c}{ Componente } & Efecto \\
\hline Salarios & 26,42 \\
Ingreso por negocios & 39,36 \\
Rentas & $-14,38$ \\
Transferencias & 19,14 \\
Pensiones & $-1,05$ \\
Remesas & 9,23 \\
Oportunidades & 10,95 \\
Otros ingresos & 0,05 \\
Ingresos de capital & 29,42 \\
Total & 100,00 \\
Cambio en el índice de Piesch & $-0,0415213$ \\
\hline
\end{tabular}

Fuente: cálculos a partir de los microdatos (datos hogar por hogar) de la Encuesta Nacional de Ingresos y Gastos de los Hogares (Inegi, 2000 y 2012). 
a) El componente más importante en la reducción de la desigualdad es el ingreso por negocios (más de 34\%).

b) Los ingresos por salarios contribuyen con una cuarta parte de la reducción de la desigualdad. En la medida en que se apliquen políticas activas que incentiven la elevación de los salarios, este componente puede aumentar su contribución para disminuir la desigualdad en México.

c) Los ingresos por rentas de los hogares están afectando negativamente la distribución del ingreso.

d) Tanto los "programas públicos tipo Oportunidades" como las remesas desempeñan un papel muy importante para la reducción de la desigualdad con que se distribuye el ingreso de los hogares en México.

\subsection{Estructura de la distribución del ingreso por componentes en México, 2000-2012}

Del análisis desarrollado hasta aquí se desprende que son los componentes de ingresos por negocios, salarios, remesas y "programas tipo Oportunidades” los que más han contribuido a la disminución de la desigualdad en la distribución de los ingresos per cápita de los hogares en México. Esta sección tiene por objetivo mostrar cómo ha evolucionado la distribución, por deciles de hogares, de esos componentes.

Los cuadros 10 y 11 muestran la estructura porcentual de la distribución del ingreso por componentes en México, en 2000 y 2012, respectivamente. La primera columna contienen los percentiles de hogares (desde 1\% que incluye a la población con más ingresos; hasta los 10\% más pobres). ${ }^{5}$ Las columnas adicionales muestran el porcentaje del ingreso de cada componente con que se queda cada percentil de la distribución. Así, por ejemplo, $1 \%$ de los hogares más ricos tenían el 9,36\% de los sueldos y salarios; $32,18 \%$ de los ingresos por rentas y $14,55 \%$ de los ingresos por pensiones en el 2012.

En el cuadro 10 se puede observar que en el 2000, 41,02\% de los ingresos por transferencias quedaban en manos del decil más rico de la población, $10 \%$ superior. De los ingresos por negocios, ese mismo estrato de

\footnotetext{
${ }^{5}$ Tanto para esta sección como para el apartado 4.1. se desarrollaron programas de cómputo que, a partir de las bases de microdatos originales (datos hogar por hogar) y tomando en cuenta el factor de expansión (ya que se trata de una muestra representativa para todo el país); permiten construir los percentiles de la distribución del ingreso desde $1 \%$ más rico hasta los $10 \%$ más pobres. Dichos programas de cómputo permitieron, además, calcular todas las medidas de desigualdad aquí empleadas así como las razones de concentración, que equivalen a calcular un "pseudo índice de Gini” para todos los componentes del ingreso pero ordenados por el ingreso total.
} 
la población tenía 47,92\%; de las pensiones 55,95\%; de los ingresos por remesas, 27,35\%; asimismo, 76,16\% de las percepciones de capital y $39,32 \%$ de los ingresos salariales. Constatamos, con los resultados presentados en el cuadro 12 que esos porcentajes se modificaron quedando de la siguiente manera: ingresos por transferencias, $38,39 \%$ (2,63\% menos que en el 2000); ingresos por negocios, $29,18 \%$ (18,74\% menos); ingresos por pensiones, 55,69\% (prácticamente no cambió); ingresos por remesas, 20,14\% (7,21\% menos); percepciones de capital, 60,58\% (12,58\% menos que en el 2000) e ingresos salariales, $37,24 \%$ (2,07\% menos).

Analizando detalladamente la evolución de todos los componentes del ingreso para todos los deciles, encontramos una regularidad: mientras que el último decil (el de los más ricos) disminuye su participación en todos los componentes de ingreso, los deciles 1 al 6 (los de los más pobres) ven aumentar el porcentaje de ingreso que tienen para prácticamente todos los componentes del ingreso total.

Por otro lado, esos cuadros permiten apreciar que los primeros cinco deciles (donde se encuentra la mayor parte de los pobres de México) tenían en el 2000,26,12\% de los ingresos por remesas, y dicho porcentaje creció a $29,57 \%$ en 2012 . Sin embargo, es notable que la mayor parte de los ingresos por remesas las tienen los deciles seis a nueve de la distribución, quienes en el 2000 tenían 46,53\% de esos ingresos y subieron su participación a 50,29\% en el 2012. Es decir, los realmente beneficiarios de las remesas en México no son los más pobres, sino ese segmento de la población a la que podemos denominar "clase media-baja". ${ }^{6}$

Lo que sí se puede constatar, en relación con los ingresos de los estratos más pobres, es que los primeros cinco deciles de hogares tenían 64,65\% de los ingresos por "programas tipo Oportunidades" en el 2000, porcentaje que subió a $67,25 \%$ en 2012 .

Finalmente, es notable observar que los ingresos del primer decil (los $10 \%$ más pobres) vía remesas cayeron (de $3,15 \%$ a $2,18 \%$ del total) y los ingresos relativos de ese segmento de población vía "programas tipo Oportunidades" también cayeron para ellos (al pasar de 24,95\% a 16,68\% en el periodo); mientras que en el extremo superior, los $10 \%$ más ricos aumentaron su participación en el ingreso vía "programas públicos tipo Oportunidades" (de 0,00\% a 0,64\% del ingreso de ese componente en el periodo). Es decir, la expansión de los programas sociales en México están llevando a un "error de focalización", en el sentido de que los pro-

${ }^{6}$ Como se puede apreciar en el cuadro 2, el ingreso promedio de lo que aquí llamamos "clase media-baja" fue, en el 2012, de la siguiente manera en dólares americanos: decil 6: 143,75; decil 7: 178,97; decil 8: 232,21 y, decil 9: 327,64. Esos montos equivalen, con un tamaño de hogar promedio de 3,9 personas y un tipo de cambio del año y mes de levantamiento de la ENIGH de 12,24 pesos por dólar; a: decil 6: 6,862; decil 7: 8,543 por hogar; decil 8: 11,085 y decil 9: 15,640 pesos mensuales. 


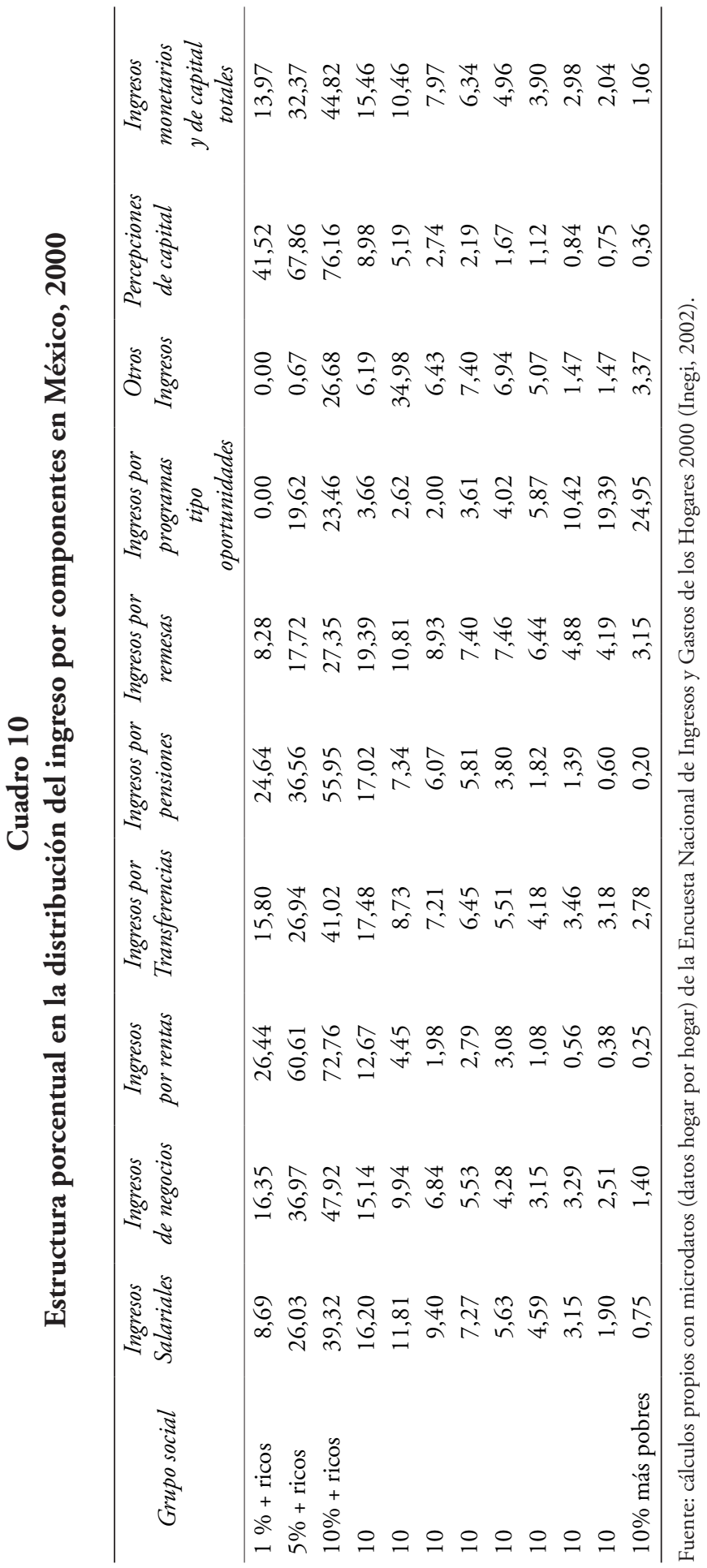




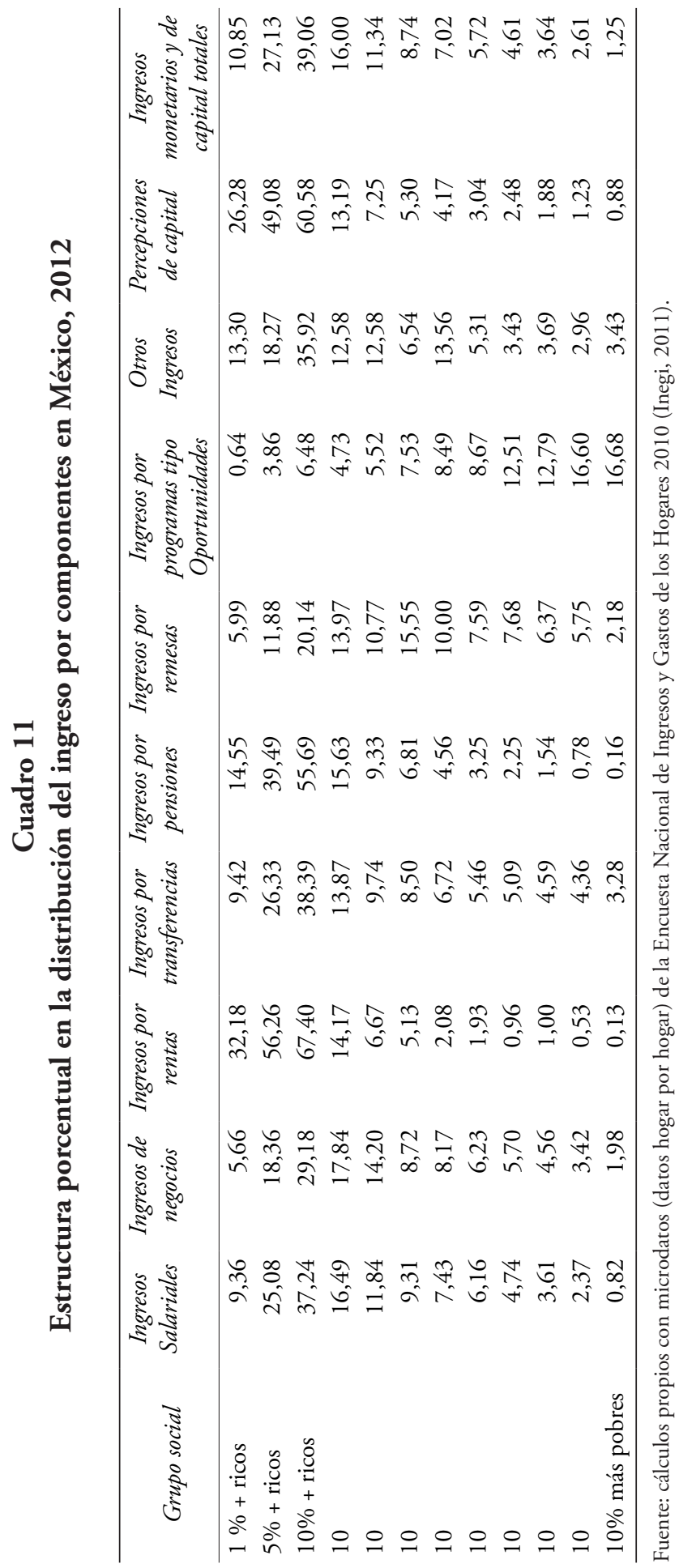


blemas de administración asociados a las dimensiones del programa probablemente sean la causa de que alguna parte de los recursos destinados para pobres, están llegando a los más ricos. Estas modificaciones estructurales explican a qué se debe que cayeron todas las medidas de desigualdad en la distribución del ingreso cuantificadas en este estudio.

\section{Conclusiones}

La disminución de la desigualdad en la distribución personal del ingreso en México, entre 2000 y 2012, se debe a fuerzas económicas que tuvieron como resultado que el ingreso relativo de los más ricos haya caído, mientras que el ingreso de los extremadamente pobres subió ligeramente. De un lado, la crisis económica internacional de 2008 y 2009 afectó los ahorros, inversiones y ganancias de los estratos más ricos de la población mientras que, por otra parte, la precaria situación de los extremadamente pobres los llevó a depender cada vez más de los programas públicos y de las remesas que reciben de sus familiares en Estados Unidos.

El índice de Mehran, que es más sensible a lo que pasa en el extremo inferior de la distribución, muestra que las remesas explican alrededor de $17 \%$ de la disminución de la desigualdad en el periodo analizado y los ingresos vía "Oportunidades" explican 14\% de la caída en la desigualdad. ${ }^{7}$ Nuestras conclusiones sugieren, por lo tanto, la necesidad de ampliar y fortalecer el Sistema de Protección Social en México.

En este estudio se aplicó la descomposición de tres importantes índices de desigualdad: Gini, Mehran y Piesch, en la explicación de la caída en el grado de desigualdad en México, entre 2000 y 2012. Uno de los hallazgos más relevantes de esta investigación es el importante efecto que dos componentes del ingreso de los hogares están teniendo para la disminución de la desigualdad: las remesas y los "programas públicos tipo Oportunidades”. De la reducción en la desigualdad total en México, alrededor de $20 \%$, se debe al efecto positivo que las remesas están teniendo. En relación con los programas públicos, mayores esfuerzos de focalización deberán ser desplegados, en virtud de que un componente importante y creciente del ingreso que generan los programas públicos está fluyendo hacia los sectores de más altos ingresos, lo que puede considerarse un efecto indeseable de la política pública.

${ }^{7}$ La relevancia de "Oportunidades" en los Hogares más pobres de México se puede explicar por su cobertura: en el 2000 llegaba sólo a 2,500,000 familias mexicanas, mientras que en 2012 fueron $5,900,000$ familias beneficiadas, con un ingreso monetario directo, vía Oportunidades. El monto de la transferencia monetaria directa a los más pobres, vía Oportunidades, se ajusta anualmente con el Índice Nacional de Precios al Consumidor. Y como aumenta con el número de hijos, los extremamente pobres que tienen más hijos reciben un beneficio mayor. 
Desde el punto de vista del desarrollo regional y territorial, parece relevante al menos dejar apuntado un hecho: en la medida en que los ingresos de las familias más pobres, tanto de las beneficiarias de programas de transferencias monetarias condicionadas como "Oportunidades (ahora Prospera)", como los ingresos de las familias que reciben remesas de familiares en Estados Unidos se distribuyen heterogéneamente, en esa medida, las posibilidades de impulsar el mercado interno serán extremadamente diferenciadas a lo largo y ancho del país.

Familias de regiones del país con una alta tasa de migración estarán recibiendo, en mayor proporción los ingresos provenientes de las remesas. De igual manera, comunidades con alto grado de marginación y pobreza están recibiendo más ingresos de "programas tipo Oportunidades". En teoría, estas tendencias pueden hacer pensar tanto en remesas como en transferencias gubernamentales como un elemento igualador entre regiones del país; pero ésta es más bien una nueva línea de investigación abierta a partir del presente estudio.

Un hecho relevante que debemos destacar en esta investigación es que, mientras que la desigualdad en la distribución personal del ingreso cayó en México entre 2000 y 2012, la pobreza extrema aumentó. La explicación de la caída en la desigualdad ya la hemos dado (aumento del ingreso de los más pobres y caída simultanea del ingreso de los más ricos, por la crisis). Pero el aumento de la pobreza tiene su origen, básicamente, en el estancamiento de los salarios y en la falta de generación de empleos. Como se sabe, las familias más pobres dependen de manera crucial de los ingresos por trabajo. ¿Qué pasó con el ingreso por trabajo de los más pobres?

Esta investigación muestra que los ingresos por sueldos y salarios, a pesar de constituir la parte más relevante del ingreso total de los hogares (65\% en 2012), no contribuyen a disminuir la desigualdad en esa misma proporción: para todos los índices aquí estudiados, el factor trabajo apenas logró explicar poco menos de $30 \%$ de la disminución en la desigualdad (29,35\% vía índice de Gini; $28,66 \%$ vía índice de Mehran y 26,42\% vía índice de Piesch). Otras investigaciones (Aguilar-Gutiérrez, 2012 y 2009) muestran que la pobreza, en el mismo periodo, aumentó. Paradójicamente, entonces, la desigualdad cayó y la pobreza creció; pero si la pobreza creció, la explicación está en la evolución del empleo y de los salarios de los más pobres: la tasa de crecimiento promedio anual de la economía en México, entre 2001 y 2012 fue de apenas 1,9\%, crecimiento que apenas puede generar alrededor de 335,000 empleos al año ${ }^{8} \mathrm{y}$, por

\footnotetext{
${ }^{8}$ De acuerdo con Aguilar-Gutiérrez (2009) en un análisis de Elasticidad-Empleo del Ingreso, por cada punto porcentual que crece la economía mexicana, se generan alrededor de 176,500 empleos nuevos.
} 
lo tanto, crecimiento económico insuficiente para generar los puestos de trabajo que necesitan los más pobres.

Por lo tanto, con fines de políticas públicas, se deduce que una política salarial activa, que contribuya a la recuperación de los niveles salariales de los sectores más pobres de la población, es necesaria en México, con la finalidad de que esa fuente de ingresos cumpla con su función clásica de distribución progresiva de los ingresos. Pero también mayores tasas de crecimiento económico, capaces de proveer de fuentes permanentes de ingreso a los más pobres.

\section{Fuentes consultadas}

Aguilar-Gutiérrez, Genaro (2012), "Tres décadas de desigualdad y erosión de la clase media en México", Cadernos PROLAM, 11 (21), Universidade de São Paulo, São Paulo, pp. 93-112.

Aguilar-Gutiérrez, Genaro (2009), ¿Puede el crecimiento económico eliminar la pobreza?, Universidad Nacional Autónoma de México, México.

Araar, Abdelkrim (2004), "On the decomposition of the Gini index: An exact approach”, Working Paper 06-02, Centre Interuniversitaire sur le Risque, les Politiques Économiques et l'Emploi, Montreal, pp. 1-25.

Aristondo, Oihana, José Luis García Cassilda Lasso de la Vega y Ricardo Alberto Marques (2011), "The Gini index, the dual decomposition of aggregation functions; and the consistent measurement of inequality", International Journal of Intelligent Systems, 27 (2), Wiley, pp. 132-152.

Azevedo Joao Pedro, Gabriela Inchaust y Viviane Sanfelice (2013), "Decomposing the recent inequality decline in Latin America", Policy Research, Working Paper No. 6715, World Bank, Washington, pp. 1-26.

Chantreuil, Fréderic y Alain Trannov (2013), "Inequality decomposition values: the trade-off between marginality and efficiency", The Journal of Economic Inequality, 11 (1), Springer, New York, pp. 83-98. 
Chen, Jiandong, Dai Dai, Ming Pu, Wenxuan Hou y Qiaobin Feng (2010), "The trend of the Gini coefficient of China", Brooks World Poverty Institute, Worwing Paper No. 109, The University of Manchester, Manchester, pp. 1-32.

Cowell, Frank y Carlo Fiorio (2006), "Rethinking inequality decomposition: comment", London School of Economics, London, pp. 1-8.

Cowell, Frank (2008), Measuring Inequality, Oxford University Press, Oxford.

Cowell, Frank (1988), "Inequality decomposition: three dad measures", Bulletin of Economic Research, 40 (4), Wiley-Blackwell, pp. 309-312.

Esquivel, Gerardo, Nora Lustig y John Scott (2010), "A decade of falling inequality in Mexico: market forces or state action?”, Discussion Paper, United Nations Development Programme, New York, pp. 175-217.

Foster, James, Joel Greer and Erik Thorbecke Source (1984), "A class of decomposable poverty measures", Econometrica, 52 (3), The Econometric Society Stable, pp. 761-766.

Giovanni, Bellú Lorenzo (2006), "Decomposition of income inequality by income sources", Policy Impacts on Inequality, Organización de las Naciones Unidas para la Alimentación y la Agricultura, Roma, pp. 1-14.

Griffiths, William (2008), "On dagum's decomposition of the Gini coefficient", Research Paper No. 1054, The University of Melbourne, Melbourne, pp. 1-9.

Hoffmann, Rodolfo (2009), "Inequality of income distribution in Brazil: the contribution of pensions and of other components of per cápita household income", Economia e Sociedade, 18 (1), UNICAMP, São Paulo.

Inegi (Instituto Nacional de Estadística y Geografía) (2013), "Encuesta Nacional de Ingresos y Gastos de los Hogares de México-Base de datos", Inegi, México. 
Inegi (Instituto Nacional de Estadística y Geografía) (2012), "Encuesta Nacional de Ingresos y Gastos de los Hogares de México-Base de datos", Inegi, México.

Inegi (Instituto Nacional de Estadística y Geografía) (2002), "Encuesta Nacional de Ingresos y Gastos de los Hogares de México-Base de datos", Inegi, México.

Inegi (Instituto Nacional de Estadística y Geografía) (2000), “Encuesta Nacional de Ingresos y Gastos de los Hogares de México-Base de datos", Inegi, México.

Inegi (Instituto Nacional de Estadística y Geografía) (1996), "Encuesta Nacional de Ingresos y Gastos de los Hogares de México-Base de datos", Inegi, México.

Kakwani, Nanak y Ernesto Pernia (2000), “What is pro-poor growth?”, Asian Development Review, 18 (1), Asian Development Bank, Manila, pp. 1-16.

Levy, Santiago (2010), Buenas intenciones, malos resultados, Política social, informalidad y crecimiento económico en México, Oceano, México.

Mussard, Stéphane, Seyte Françoise y Terraza Michel (2003), "Decomposition of Gini and the generalized entropy inequality measures", Economics Bulletin, 4 (7), Editorial Board, Elsevier, pp. 1-6.

Pyatt, Graham (1976), "On the interpretation and disaggregation of Gini coefficient", The Economic Journal, 86 (342), Royal Economic Society, pp. 243-255.

Pyatt, Graham, Chau-Nan Chen y Jhon Fei (1980), "The distribution of income by factor components", The Quarterly Journal of Economics, 95 (3), Oxford University Press, Oxford, pp. 451-473.

Ravallion, Martin y Shaohua Chen (2003), “Measuring Pro-Poor Growth”, Policy Research Working Paper, World Bank, Washington, pp. 1-11.

Sen, Pranab K. y Julio M. Singer (1994), Large sample methods in statistics. An introduction with applications, Chapman \& Hall, London. 
Soares, Sergei (2006), "Distribuição de renda no Brasil de 1976 a 2004 com ênfase no período entre 2001 e 2004", texto para Discussão, núm. 1166, IPEA, Brasilia, pp. 1-31.

Shorrocks, Anthony F. (2013), "Decomposition procedures for distributional analysis: a unified framework based on the Shapley value", The Journal of Economic Inequality, 11 (1), Springer, pp. 99-126.

Shorrocks, Anthony F. (1982), "Inequality decomposition by factor components", Econometrica: Journal of the Econometric Society, 50 (1), New York, pp. 193-211.

Shorrocks, Anthony F. (1980), "The class of additively decomposable inequality measures", Econometrica: Journal of the Econometric Society, 48 (3), New York, pp. 613-625.

Son, Hyun Hwa (2004), "A note on pro-poor growth", Journal of Economics Letters, vol. 82, Elsevier, New York, pp. 307-314.

Van Kerm, Philippe (2009), Generalized Gini and concentration coefficients with factor decomposition, DRAPS 1293, CEPS/INSTEAD, Luxemburg.

Recibido: 21 de septiembre de 2014. Corregido: 21 se septiembre de 2015. Aceptado: 11 de octubre de 2015.

Genaro Aguilar-Gutiérrez. Mexicano. Doctor en ciencias económicas por la Universidad Estadual de Campinas de São Paulo, Brasil. Es miembro del Sistema Nacional de Investigadores, nivel I. Actualmente, es profesor en el doctorado de la sección de estudios de posgrado e investigación, Escuela Superior de Economía del Instituto Politécnico Nacional. Su línea de investigación incluye temas de desigualdad, pobreza, mercados laborales y política social. Entre sus últimas publicaciones destacan, en coautoría: "Análisis del comportamiento estadístico y aproximación fractal en la recaudación del impuesto sobre nóminas y asimilados en el estado de Quintana Roo", Contaduría y Administración, 59 (4), UNAM, México, pp. 71-86 (2014); Tres décadas de desigualdad y erosión de la clase media en México, Brazilian Journal of Latin American Studies, 11 (2), São Paulo, pp. 93-112 (2012); Desigualdady Pobreza en México 19842010: ¿Deterioro de la situación social en México?, 10 (19), São Paulo, pp. 31-59 (2011). 